\title{
Periventricular Region
}

National Cancer Institute

\section{Source}

National Cancer Institute. Periventricular Region. NCI Thesaurus. Code C132390.

The area surrounding the ventricles of the brain. 